\title{
ORFEUS OBSERVATIONS OF ULTRAVIOLET EXCITED HIGH-J MOLECULAR HYDROGEN
}

\author{
Dae-Hee Lee ${ }^{1}$ W. Van Dyke Dixon ${ }^{2}$, Kyoung-Wook Min ${ }^{3}$, and Soojong PaK ${ }^{4}$ \\ ${ }^{1}$ Korea Astronomy and Space Science Institute, Daejeon 305-348, Korea \\ E-mail: dhlee@kasi.re.kr \\ ${ }^{2}$ Department of Physics and Astronomy, The Johns Hopkins University, Baltimore, Maryland 21218, USA \\ E-mail:wvd@pha.jhu.edu \\ ${ }^{3}$ Korea Advanced Institute of Science and Technology, Daejeon 305-701, Korea \\ E-mail:kwmin@space.kaist.ac.kr \\ ${ }^{4}$ Department of Astronomy and Space Science, Kyung Hee University, Gyeonggi-do 446-701, Korea \\ E-mail: soojong@khu.ac.kr
}

(Received November 02, 2009; Accepted November 20, 2009)

\begin{abstract}
We present measurements of diffuse interstellar $\mathrm{H}_{2}$ absorption lines in the continuum spectra of 10 early-type stars. The data were observed with the Berkeley Extreme and Far-Ultraviolet Spectrometer (BEFS) of the ORFEUS telescope on board the ORFEUS-SPAS I and II space-shuttle missions in 1993 and 1996, respectively. The spectra extend from the interstellar cutoff at $912 \AA$ to about $1200 \AA$ with a resolution of $\sim 3000$ and statistical signal-to-noise ratios between 10 and 65 . Adopting Doppler broadening velocities from high-resolution optical observations, we obtain the $\mathrm{H}_{2}$ column densities of rotational levels $J^{\prime \prime}=0$ through 5 for each line of sight. The kinetic temperatures derived from $J^{\prime \prime}=0$ and 1 states show a small variation around the mean value of $80 \mathrm{~K}$, except for the component toward HD 219188, which has a temperature of $211 \mathrm{~K}$. Based on a synthetic interstellar cloud model described in our previous work, we derive the incident UV intensity $I_{U V}$ and the hydrogen density $n_{H}$ of the observed components to be $-0.4 \leq \log I_{U V} \leq 2.2$ and $6.3 \leq n_{\mathrm{H}} \leq 2500 \mathrm{~cm}^{-3}$, respectively.
\end{abstract}

Key words : ISM: $\mathrm{H}_{2}$ — ISM: molecular clouds — ultraviolet: FUV

\section{INTRODUCTION}

The hydrogen molecule $\left(\mathrm{H}_{2}\right)$ plays a central role in a variety of processes that significantly influence the chemical and physical state of the interstellar medium (ISM). From the Copernicus satellite (Savage et al. 1977 ) to the Far Ultraviolet Spectroscopic Explorer (FUSE) (Shull et al. 2000), many systematic measurements of $\mathrm{H}_{2}$ absorption in the far-ultraviolet (FUV) have derived $N(J)$, the column density of $\mathrm{H}_{2}$ in the rotational level $J$ of the ground vibrational and electronic state, to obtain physical conditions of the diffuse and translucent molecular clouds in the ISM (see references in Lee, Pak, Dixon, \& van Dishoeck 2007, hereafter Paper I). Recently, detailed $\mathrm{H}_{2}$ models have been used to obtain constraints on otherwise unobservable parameters of the molecular clouds by reproducing the observed $N(J)$ terms (Browning et al. 2003; Paper I). For example, in Paper I we found that $N(4) / N(0)$ is proportional to the incident UV intensity $I_{\mathrm{UV}}$ and the $\mathrm{H}_{2}$ molecular fraction $f$ is simply related to the ratio of $I_{\mathrm{UV}}$ and the hydrogen density $n_{\mathrm{H}}$, providing a new method to derive $I_{\mathrm{UV}}$ and $n_{\mathrm{H}}$ from the observational parameters $N(4) / N(0)$ and $f$.

Corresponding Author: D.-H. Lee
In this paper, we present results from the molecularhydrogen survey conducted by the Berkeley Extreme and Far-Ultraviolet Spectrometer (BEFS) on the ORFEUS telescope. Since the BEFS has moderate spectral resolution, we have used high-resolution optical measurements to select 10 target stars with a single absorption component for our study. From the column densities of each rotational level $N(J)$, we derive the total hydrogen column density $N\left(\mathrm{H}_{2}\right)$, the cloud mean temperature $T_{01}$, and the excitation temperature $T_{\text {ex }}$. We use the relation between $N(4) / N(0)$ and $f$ to derive $I_{\mathrm{UV}}$ and $n_{\mathrm{H}}$ for each line of sight as described in Paper I. Combining our data with new observational results from FUSE (Gillmon et al. 2006), we explore the relation between $n_{\mathrm{H}}$ and $N\left(\mathrm{H}_{2}\right)$ in interstellar molecular clouds.

\section{OBSERVATIONS AND ANALYSIS}

The BEFS flew on board the ORFEUS-SPAS I and II Space Shuttle missions in 1993 and 1996, respectively. Its performance on the first mission is described by Hurwitz \& Bowyer (1995) and on the second by Hurwitz et al. (1998). Its spectral resolution at FUV wavelengths is $\sim 95 \mathrm{~km} \mathrm{~s}^{-1}$ FWHM $(\sim 0.33 \AA)$ for point sources. Its effective area peaked at about $4 \mathrm{~cm}^{2}$ 
TABLE 1

TARget Summary

\begin{tabular}{|c|c|c|c|c|c|c|c|}
\hline Star & $l$ & $b$ & Sp. Type & $E(B-V)$ & $\log N(\mathrm{HI})\left[\mathrm{cm}^{-2}\right]$ & $d[\mathrm{pc}]$ & References* \\
\hline HD 36402 & 277.77 & -33.04 & $\mathrm{OB}+\mathrm{WC} 5$ & 0.11 & 20.72 & 52500 & SB81, W95, SDS93 \\
\hline HD 94473 & 272.83 & +29.17 & B3.0 IV & 0.14 & 20.89 & 278 & HIP, P92, P93 \\
\hline HD 97991 & 262.34 & +51.73 & $\mathrm{~B} 2.0 \mathrm{~V}$ & 0.01 & 20.64 & 824 & A83, DS94, A83 \\
\hline HD 149881 & 31.38 & +36.23 & B0.5 III & 0.07 & 20.62 & 2139 & DS94, SF95, S98 \\
\hline HD 156110 & 70.99 & +35.71 & B3 Vn & 0.03 & 20.66 & 567 & F94 \\
\hline HD 167756 & 351.47 & -12.30 & $\mathrm{~B} 0.5 \mathrm{Ia}$ & 0.10 & 20.81 & 4000 & DS94, H97, KBK98 \\
\hline HD 203664 & 61.93 & -27.46 & $\mathrm{~B} 0.5 \mathrm{~V}$ & 0.03 & 20.54 & 3200 & F94, SM87 \\
\hline HD 214080 & 44.80 & -56.92 & B1.0 Ib & 0.05 & 20.61 & 3381 & DS94, H97, SDS93 \\
\hline HD 219188 & 83.03 & -50.17 & B0.5 III & 0.08 & 20.75 & 2379 & F94, KDM82 \\
\hline HD 220582 & 99.41 & -33.38 & B6 IV,V & 0.07 & 20.5 & 292 & HIP, O87, S66 \\
\hline
\end{tabular}

${ }^{*}$ References. - A83: Albert 1983; DS94: Diplas \& Savage 1994a; F94: Fruscione et al. 1994; H97: Howarth et al. 1997; HIP: ESA 1997; O87: Oja 1987; S74: Sharpless 1974; SF95: Spitzer \& Fitzpatrick 1995; S66: Slettebak 1966; KDM82: Keenan et al. 1982; SM87: Savage \& Massa 1987; P92: Penprase 1992; SB81: Savage \& de Boer 1981; W95: Walborn et al. 1995; SDS93: Sembach et al. 1993; S98: Sembach (private communication); P93: Penprase 1993; W97: Welsh et al. 1997; KBK98: Kennedy et al. 1998

TABLE 2

Observation and Analysis Summary

\begin{tabular}{ccrlllll}
\hline \hline Star & Mission & Time $^{\mathrm{a}}[\mathrm{s}]$ & $\mathrm{S} / \mathrm{N}^{\mathrm{b}}$ & Region Fit $[\AA]$ & $\langle v\rangle^{\mathrm{c}}\left[\mathrm{km} \mathrm{s}^{-1}\right]$ & $b^{\mathrm{c}}\left[\mathrm{km} \mathrm{s}^{-1}\right]$ & Continuum $^{\mathrm{d}}$ \\
\hline HD 36402 & I & 1306 & 16.6 & $1045-1058$ & +11.0 (SDS 93) & 3 (SDS 93) & HD 37018 \\
HD 94473 & II & 288 & 21.2 & $1088-1102$ & $-3.4($ P93) & 2 (P93) & HD 155763 \\
HD 97991 & II & 500 & 39.3 & $1088-1101.5$ & -5.7 (A83) & 4 (A83) & HD 37018 \\
HD 149881 & I & 1560 & 52.3 & $1045-1060$ & +1.1 S98) & 2 (S98) & HD 122451 \\
HD 156110 & I & 840 & 48.3 & $1045-1060$ & -1.7 (D00) & 3 (D00) & HD 158408 \\
HD 167756 & I & 866 & 51.0 & $1045-1060$ & +3.5 (KBK98) & 3 (KBK98) & HD 38771 \\
HD 203664 & II & 652 & 40.9 & $1088-1102$ & +1.8 (D00) & 4 (D00) & HD 122451 \\
HD 214080 & I & 1550 & 61.1 & $1045-1060$ & -5.4 (SDS93) & 2 (SDS93) & HD 91316 \\
HD 219188 & II & 186 & 25.6 & $1088-1102$ & -3.7 (D00) & 6 (D00) & HD 36486 \\
HD 220582 & II & 1083 & 28.5 & $1088-1102$ & -8.8 (D00) & 4 (D00) & HD 155763 \\
\hline
\end{tabular}

a Total integration time.

bStatistical signal-to-noise ratio in a $0.25 \AA$ bin, averaged over the region fit.

${ }^{\mathrm{c}}$ Obtained from Na I optical observations. See references.

${ }^{\mathrm{d}}$ Copernicus continuum stars (Snow \& Jenkins 1977).

*References. - A83: Albert 1983; P93: Penprase 1993; SDS93: Sembach et al. 1993; S98: Sembach (private communication); KBK98: Kennedy et al. 1998; D00: Dixon (private communication) 
for the first mission and about $9 \mathrm{~cm}^{2}$ for the second. From the 54 early-type target stars in the Galactic disk and halo and 3 stars in the Magellanic Clouds observed by the BEFS, we have chosen 10 program stars (Table 1) with only a single major velocity component in the high-resolution optical measurements, to avoid line blending. If there are multiple components toward a star, we include it in our sample if the column density of the principal component is more than 3 times the sum of the other components. $N(\mathrm{HI})$ is important to derive the molecular fraction $f$. We referred the $N(\mathrm{HI})$ values obtained from the $21 \mathrm{~cm}$ radio observations (see the references in Table 1 ).

Table 2 shows the observation log and the summary of results. We use the optical Na I absorption features to obtain the Doppler parameters of the principal absorption components, because the $\mathrm{Na} I$ lines trace the diffuse, cloudy component of the ISM (Sembach \& Danks 1994). Observed Doppler parameters in Table 2, rounded to the nearest $b$ value in our model grid, range from 2 to $6 \mathrm{~km} \mathrm{~s}^{-1}$, consistent with values found by other authors who use Na I lines to estimate Doppler parameters (e.g., Sonnentrucker et al. 2003). To estimate the stellar continuum, we use reference spectra selected from the Copernicus Spectral Atlas (Snow \& Jenkins 1977). Reference spectra, listed in Table 2, are selected for their similarity to the observed stars in spectral classification. Priority is given to the shape of the stellar continuum, and we seek the best match among the reference stars of similar spectral type. Because the total $\mathrm{H}_{2}$ column density $N\left(\mathrm{H}_{2}\right)$ in each reference spectrum is less than the uncertainty in either the $J^{\prime \prime}=0$ or $J^{\prime \prime}=1$ column of the corresponding program star, we do not attempt to correct for $\mathrm{H}_{2}$ absorption in the reference spectrum. The atlas spectra have $0.2 \AA$ resolution, close to that of our data.

We begin our analysis by generating a set of synthetic $\mathrm{H}_{2}$ absorption-line models, using an ISM linefitting package written by M. Hurwitz and V. Saba. Given the column density and Doppler broadening parameter, the program computes a Voigt profile for each absorption feature and outputs a spectrum of $\tau$ versus wavelength at a resolution equal to the pixel size $(0.0142 \AA)$ of the BEFS spectra (which are oversampled). The high resolution is necessary because the $\mathrm{H}_{2}$ absorption lines are intrinsically quite narrow. For each $\mathrm{H}_{2}$ rotational level $\left(J^{\prime \prime}=1-5\right)$, we produce a set of absorption-line spectra with the given Doppler parameters.

The models are fitted to the data using the nonlinear curve-fitting program SPECFIT (Kriss 1994) to perform a $\chi^{2}$ minimization. For stars observed on the 1993 ORFEUS-SPAS I flight, we fit the spectral region between 1045 and $1060 \AA$, which contains most of the $v=0 \rightarrow 4$ vibrational band of the Lyman series, as this region is least complicated by stellar photospheric absorption or overlapping Werner bands of interstellar $\mathrm{H}_{2}$. Unfortunately, the brightest stars observed on the 1996 flight exhibit detector artifacts in this part of the spectrum (Hurwitz et al. 1998), so we use the 1088-1102 $\AA$ region, which contains the $v=0 \rightarrow 1$ Lyman series vibrational band, for all but the faintest ORFEUS-SPASII stars. The exact region fitted for each of our program stars is listed in Table 2. For all stars, only the $J^{\prime \prime}=0$ to 5 rotational features are considered. Photoabsorption to levels with $J^{\prime \prime}>6$ immediately cascade to the $J^{\prime \prime}=5$ or $J^{\prime \prime}=6$ level for ortho and para $\mathrm{H}_{2}$, respectively (Jura 1974), and $J^{\prime \prime}=6$ lines are rarely observed. We model each $\mathrm{H}_{2}$ rotational level independently, using the value of the Doppler parameter $b$ from Table 2 . The radial velocities of each rotational level are tied together, but the entire absorption-line complex is allowed to shift in velocity space to account for wavelength offsets and redshifts.

We first fit the $J^{\prime \prime}=0$ and 1 lines, then add the higher-level lines, as they often lie in the wings of the lower- $J^{\prime \prime}$ features. Two interstellar features that fall in the $1045-1060 \AA$ region, Ar I at $1048.22 \AA$ and Fe II at $1055.26 \AA$, and one in the $1088-1102$ region, Fe II at $1096.88 \AA$, are also included in the model. A band of Cr III absorption features near $1100 \AA$ (Rogerson \& Ewell 1985) complicates the fit to the $\mathrm{P}(3)$ and $\mathrm{R}(4)$ lines at 1099.8 and $1100.2 \AA$, respectively, but other nearby $J^{\prime \prime}=3$ and 4 features allow us to constrain the column densities of these rotational levels. The only free parameters in the fit are the continuum placement (normalization and "tilt"), the scaling of the absorption-line column densities, and a constant shift in the wavelength scale of the absorption-line system. We adjust the model to fit the data as well as possible by hand, then invoke the automated $\chi^{2}$-minimization algorithm to fine-tune the fit.

Figure 1 shows the spectra of our target stars (thick solid lines), which were background-subtracted, scaled to correct for detector dead-time effects, and wavelength- and flux-calibrated as described by Hurwitz et al. (1998), the continuum spectra (thin solid lines) and our fitting results (dashed lines). Error bars are assigned to the data assuming Gaussian statistics. Hurwitz et al. (1998) find that, at the nominal instrument resolution of $0.33 \AA$, noise from detector artifacts is about $6.4 \%$ of the measured signal, so we sum in quadrature the random-error spectrum with a scaled (by 0.05) signal spectrum, representing this component of the systematic error. The flux calibration is based on in-flight observations of hot white dwarfs and is thought to be uncertain to less than $10 \%$ (Dixon et al. 1998).

\section{RESULTS AND DISCUSSION}

\section{(a) $\mathbf{H}_{2}$ Column Densities}

Column densities for each rotational level are presented in Table 3. The 1- $\sigma$ error bars are calculated by SPECFIT and represent only statistical uncertainties. Systematic errors, including line blending, both within the $\mathrm{H}_{2}$ absorption bands and with nearby interstellar 

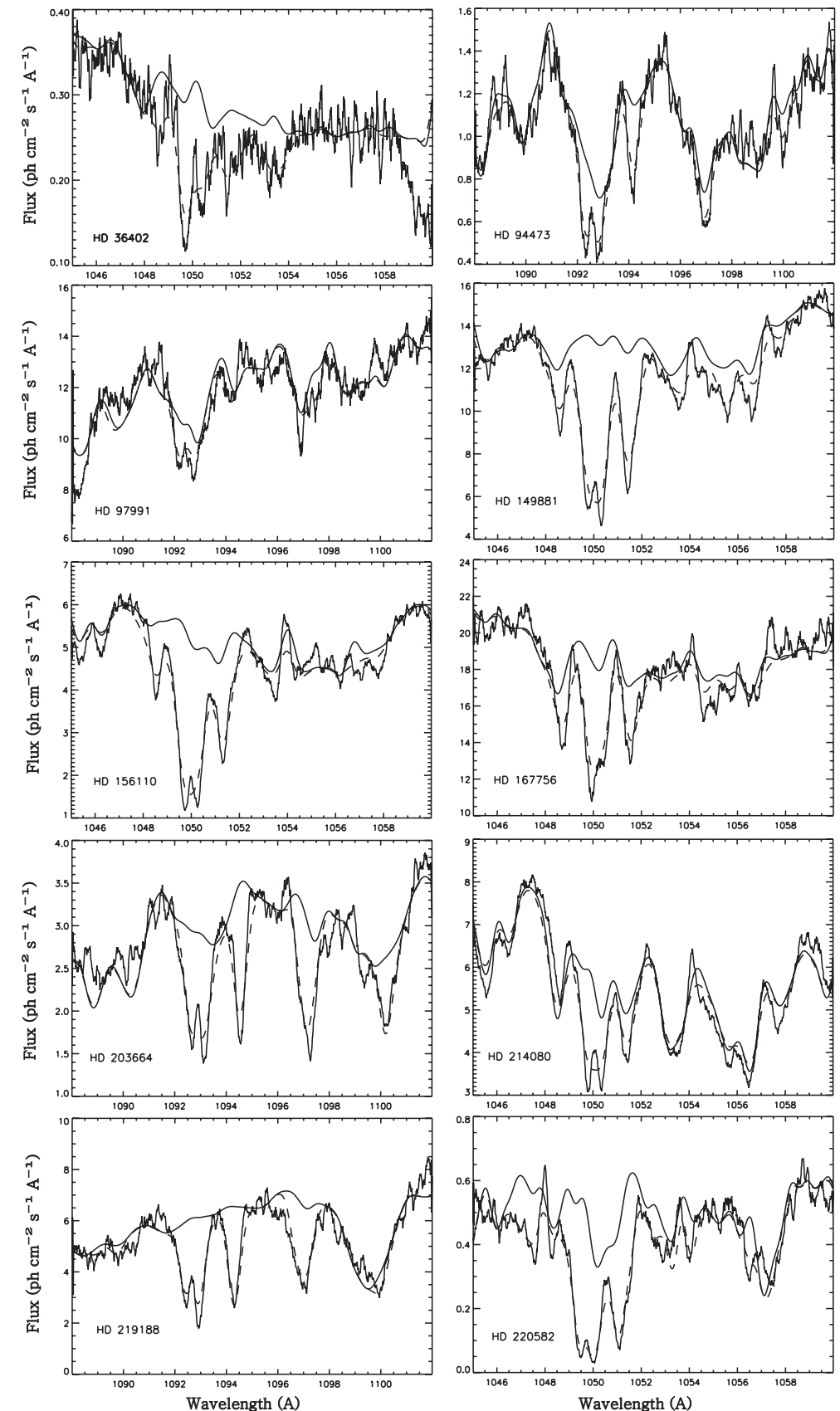

Fig. 1. - The BEFS spectra of 10 targets, binned by 9 pixels are plotted in thick solid lines. Scaled continuum spectra and the fitting results are overplotted as solid and dashed lines, respectively.

atomic lines, errors in our cloud models, and (most important) errors in our adopted stellar continua, are likely to dominate the statistical uncertainties. Because it is difficult to quantify these effects, we estimate the systematic errors from our experience working with the data. We find that, even in complicated situations, small changes in our model parameters yield changes of $30 \%$ or less in the derived column densities for $J^{\prime \prime}=0$ and 1 , and of a factor of two or less for the higher ro- tational levels, corresponding to uncertainties of $\sim 0.1$ dex for $J^{\prime \prime}=0,1$ and 0.3 dex for $J^{\prime \prime}>2$. Total column densities range from $\log N\left(\mathrm{H}_{2}\right)=17.16$ to 19.63 $\left[\mathrm{cm}^{-2}\right]$, indicating that these sight lines probe diffuse interstellar clouds.

\section{(b) Temperatures}

The mean cloud kinetic temperature $T_{01}$ for each line of sight is derived from the column densities $N(0)$ 
TABLE 3

Molecular hydrogen ROTAtional COLUMN DENSITIES

\begin{tabular}{lccccccc}
\hline \hline Star & $\log N(0)$ & $\log N(1)$ & $\log N(2)$ & $\log N(3)$ & $\log N(4)$ & $\log N(5)$ & $\log N\left(\mathrm{H}_{2}\right)$ \\
\hline HD 36402 & 18.51 & 18.10 & 15.93 & 13.59 & 13.66 & 14.73 & 18.65 \\
& \pm 0.02 & \pm 0.04 & \pm 0.15 & \pm 0.37 & \pm 1.04 & \pm 0.15 & \pm 0.02 \\
HD 94473 & 18.61 & 18.46 & 15.18 & 17.07 & 15.15 & 14.44 & 18.85 \\
& \pm 0.02 & \pm 0.04 & \pm 0.17 & \pm 0.28 & \pm 0.22 & \pm 0.53 & \pm 0.02 \\
HD 97991 & 17.50 & 16.47 & 14.62 & 14.05 & 14.09 & 13.30 & 17.54 \\
& \pm 0.14 & \pm 0.27 & \pm 0.11 & \pm 1.92 & \pm 0.44 & \pm 1.44 & \pm 0.13 \\
HD 149881 & 18.55 & 18.75 & 15.78 & 15.44 & 14.46 & 14.43 & 18.96 \\
& \pm 0.00 & \pm 0.00 & \pm 0.01 & \pm 0.01 & \pm 0.01 & \pm 0.01 & \pm 0.00 \\
HD 156110 & 18.91 & 18.79 & 15.23 & 15.08 & 14.24 & 14.18 & 19.16 \\
& \pm 0.00 & \pm 0.00 & \pm 0.00 & \pm 0.00 & \pm 0.00 & \pm 0.01 & \pm 0.00 \\
HD 167756 & 17.06 & 16.41 & 15.56 & 15.35 & 14.30 & 14.94 & 17.16 \\
& \pm 0.00 & \pm 0.00 & \pm 0.00 & \pm 0.00 & \pm 0.00 & \pm 0.00 & \pm 0.00 \\
HD 203664 & 18.29 & 18.31 & 16.31 & 18.03 & 14.08 & 12.36 & 18.71 \\
& \pm 0.05 & \pm 0.05 & \pm 0.07 & \pm 0.25 & \pm 0.80 & \pm 1.56 & \pm 0.06 \\
HD 214080 & 18.19 & 17.91 & 14.34 & 16.23 & 14.17 & 14.15 & 18.38 \\
& \pm 0.02 & \pm 0.04 & \pm 0.18 & \pm 0.15 & \pm 0.18 & \pm 0.19 & \pm 0.02 \\
HD 219188 & 18.38 & 18.99 & 16.36 & 16.36 & 15.14 & 15.03 & 19.09 \\
HD 220582 & \pm 0.07 & \pm 0.03 & \pm 0.12 & \pm 0.10 & \pm 0.15 & \pm 0.15 & \pm 0.03 \\
& 19.25 & 19.31 & 18.43 & 18.14 & 15.41 & 13.64 & 19.63 \\
& \pm 0.04 & \pm 0.02 & \pm 0.07 & \pm 0.11 & \pm 0.14 & \pm 2.45 & \pm 0.02 \\
\hline
\end{tabular}

${ }^{*}$ Units are $\mathrm{cm}^{-2}$. Quoted errors are statistical uncertainties returned by SPECFIT.

and $N(1)$ using the relation

$$
\frac{N(1)}{N(0)}=\frac{g_{1}}{g_{0}} \exp \left(\frac{-E_{01}}{k T_{01}}\right)=9 \exp \left(\frac{-170 \mathrm{~K}}{T_{01}}\right),
$$

where $g_{0}$ and $g_{1}$ are the statistical weights of $J^{\prime \prime}=0$ and $J^{\prime \prime}=1$, respectively (Shull \& Beckwith 1982). Table 4 shows that the derived temperatures range from $37 \mathrm{~K}$ for HD 97991 to $211 \mathrm{~K}$ for HD 219188 and average $T_{01}=80 \pm 11$ (rms) $\mathrm{K}$, in agreement with the Copernicus result of $T_{01}=77 \pm 17 \mathrm{~K}$ (Savage et al. 1977). Gillmon et al. (2006) also obtained $\left\langle T_{01}\right\rangle=124$ \pm 8 , suggesting that the average kinetic temperature of the high-latitude clouds is somewhat higher than that in the Galactic disk, although the range of $T_{01}$ is about the same as ours.

The excitation temperature $T_{\mathrm{ex}}$ is derived in Table 4 in the same way, with $J^{\prime \prime}=2$ and $J^{\prime \prime}=3$ levels. We have used $g_{J}=g_{S}(2 J+1)$ to get $g_{2}$ and $g_{3}$. Here, the spin factor $g_{S}$ equals 1 or 3 for para- or ortho- $\mathrm{H}_{2}$, respectively. The band energy factor $E_{23} \mathrm{k}^{-1}$ is 1162 K. $T_{\text {ex }}$ values range from 423 to $810 \mathrm{~K}$, with a mean of $603 \pm 54$ (rms) K. This value is slightly higher than that $(505 \pm 28 \mathrm{~K})$ found in the high-latitude FUSE survey (Gillmon et al. 2006), and twice that of the FUSE disk survey (Shull et al. 2005). Considering that most our sight lines probe the Galactic halo - only HD 167756 has a Galactic latitude $|b|<20^{\circ}$ - it is reasonable that our mean value of $T_{\mathrm{ex}}$ is similar to the FUSE high- latitude survey result.

\section{(c) The Hydrogen Density and The Incident UV Intensity}

In Paper I, we used synthetic interstellar cloud models to investigate the formation and destruction of high$J$ molecular hydrogen in photo-dissociation regions. We found that $N(4) / N(0)$ is proportional to the incident UV intensity $I_{\mathrm{UV}}$ and that the $\mathrm{H}_{2}$ molecular fraction $f$ is simply related to the ratio of $I_{\mathrm{UV}}$ and the hydrogen density $n_{\mathrm{H}}$, providing a new method to derive $I_{\mathrm{UV}}$ and $n_{\mathrm{H}}$ from the observational parameters $N(4) / N(0)$ and $f$, for an assumed $\mathrm{H}_{2}$ formation rate $R$. Note that the $\mathrm{H}_{2}$ formation rate $R$ can be written in its simplest form as

$$
R=3 \times 10^{-18} T^{1 / 2} y_{f},
$$

where $y_{f}$ is the formation rate coefficient, and $I_{\mathrm{UV}}$ is the enhancement factor compared with the mean interstellar value adopted by Draine (1978), i.e., $\phi(\lambda=1000$ $\AA)=4.5 \times 10^{-8}$ photons $\mathrm{cm}^{-2} \mathrm{~s}^{-1} \mathrm{~Hz}^{-1}$ when $I_{\mathrm{UV}}=$ 1.

Figure 2 shows the model calculation results for log $N(4) / N(0)$ vs. $\log f$ for three values of $n_{\mathrm{H}}$ (black plus: $10 \mathrm{~cm}^{-3}$, black asterisk: $100 \mathrm{~cm}^{-3}$, and black diamond signs: $\left.1000 \mathrm{~cm}^{-3}\right)$ using $T=100 \mathrm{~K}, I_{\mathrm{UV}}=0.1-1000$, and $\log N\left(\mathrm{H}_{2}\right)=14-19\left[\mathrm{~cm}^{-2}\right]$. Here, we assume the 


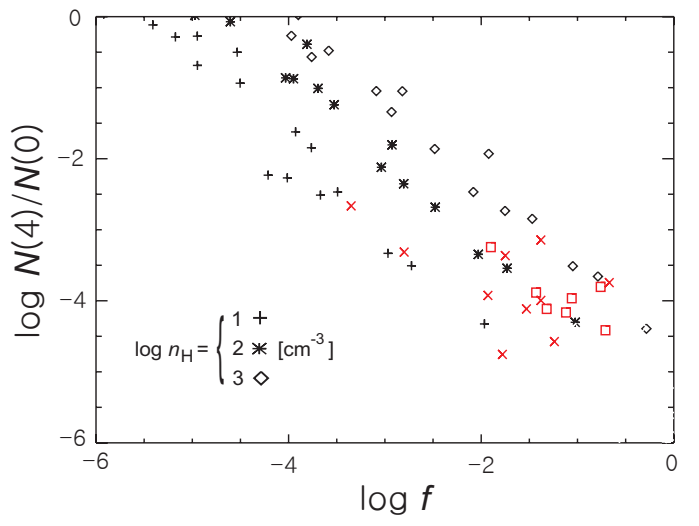

Fig. 2.- Model calculation results (see Paper I) of log $N(4) / N(0)$ vs. $\log f$ for three values of $n_{\mathrm{H}}$ (black plus: 10 $\mathrm{cm}^{-3}$, black asterisk: $100 \mathrm{~cm}^{-3}$, and black diamond signs: $1000 \mathrm{~cm}^{-3}$ ) using $T=100 \mathrm{~K}, y_{f}=1, I_{\mathrm{UV}}=0.1-1000$, and $\log N\left(\mathrm{H}_{2}\right)=14-19 \mathrm{~cm}^{-2}$. The BEFS and FUSE (Gillmon et al. 2006) observational results from Table 2 are overplotted as red $\mathrm{X}$ and red square signs, respectively.

$\mathrm{H}_{2}$ formation rate $R$ to be $3 \times 10^{-16}$, which is about 10 times the average interstellar value (see details in the Paper I). The BEFS results shown in Table 4 are plotted as red cross signs and the FUSE high-latitude survey results observed by Gillmon et al. (2006) are plotted as red squares in Fig. 2. Note that only 7 of the 45 targets in Gillmon et al. (2006) have measured $N(4)$ column densities. The models reproduce the observational results quite well.

Using these models, we have derived $I_{\mathrm{UV}}$ and $n_{\mathrm{H}}$ for our targets and the FUSE targets plotted in Fig. 2. As shown in Table 5, the derived incident UV intensity $I_{\mathrm{UV}}$ ranges from 0.4 (HD 36402) to 158 (HD 219188) times the average interstellar value. It is interesting to note that most sight lines have higher UV intensities than the average value near the Sun. The hydrogen density $n_{\mathrm{H}}$ for most sight lines in Table 5 is $\sim 100 \mathrm{~cm}^{-3}$.

Note that the smallest value is $6.3 \mathrm{~cm}^{-3}$ toward HD 36402 and the largest is $2.5 \times 10^{3} \mathrm{~cm}^{-3}$ toward HD 219188. HD 36402 is an LMC star that has been observed many times because of its interesting highvelocity absorption components around $+150 \mathrm{~km} \mathrm{~s}^{-1}$ (Savage \& de Boer 1981; Wakker 2001). In this study, we find that, beside the $11 \mathrm{~km} \mathrm{~s}^{-1}$ component, there is another low-velocity component at $0 \mathrm{~km} \mathrm{~s}^{-1}$ toward HD 36402, which is a low-density gas with a low incident UV intensity. In contrast, the low-velocity $(-3.7$ $\mathrm{km} \mathrm{s}^{-1}$ ) component in the spectrum of HD 219188 represents an active $\left(T_{01}=211 \mathrm{~K}, T_{\mathrm{ex}}=810 \mathrm{~K}\right)$, dense $\left(n_{\mathrm{H}}=2.5 \times 10^{3} \mathrm{~cm}^{-3}\right)$ region. Based on the correlation between the infrared brightness and the measured $21 \mathrm{~cm} \mathrm{H} \mathrm{I} \mathrm{emission} \mathrm{data} \mathrm{presented} \mathrm{in} \mathrm{Desert,}$ Bazell, \& Boulanger (1988), Ryu et al. (2000) indicated that the line of sight toward HD 219188 is lo-

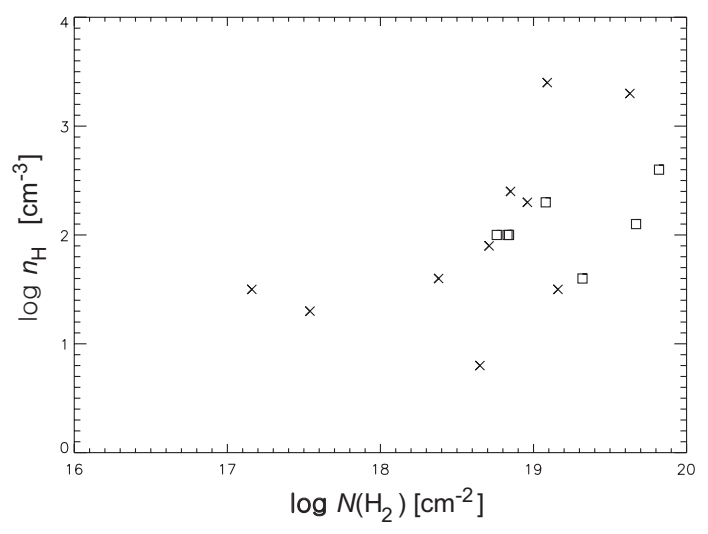

Fig. 3.- We have plotted $\log N\left(\mathrm{H}_{2}\right)$ vs. $\log n_{\mathrm{H}}$ for the BEFS (cross symbols) and the FUSE (square symbols) results from Table 3 and 5 to see the relation between the hydrogen density and the $\mathrm{H}_{2}$ column density. Only clouds with $\log N\left(\mathrm{H}_{2}\right)>18.7\left[\mathrm{~cm}^{-2}\right]$ have $n_{\mathrm{H}}>100 \mathrm{~cm}^{-3}$.

cated at the "outskirts" of the high-latitude cirrus dust clouds, which is consistent with our dense hydrogen density. There is no direct evidence of the active environment for the LV component toward HD 219188, but for reference, Welty (2007) analyzed a weak and peculiar intermediate-velocity (IV) component at -38 $\mathrm{km} \mathrm{s}^{-1}$, which has a time-variable column density.

Finally, we represent the relation between the hydrogen density and the $\mathrm{H}_{2}$ column density in Figure 3 . The BEFS and the FUSE data are plotted as cross and square symbols, respectively. Only clouds with log $N\left(\mathrm{H}_{2}\right)>18.7\left[\mathrm{~cm}^{-2}\right]$ have $n_{\mathrm{H}}>100 \mathrm{~cm}^{-3}$. This means that a minimum hydrogen density is needed to form a translucent molecular cloud, consistent with the results of Spitzer \& Jenkins (1975; see their Fig. 4). We do not compare $n_{\mathrm{H}}$ with the total hydrogen density $N(\mathrm{H})$, because the neutral hydrogen column density $N(\mathrm{H} \mathrm{I})$ includes all the hydrogen atoms along the line of sight.

\section{SUMMARY}

We present an analysis of 10 interstellar $\mathrm{H}_{2}$ absorption components observed by the BEFS during the ORFEUS-SPAS I (1995) and II (1996) missions. From the measured $\mathrm{H}_{2}$ column densities of each rotational level $N(J)$, we derive total hydrogen column densities $17.16 \leq \log N\left(\mathrm{H}_{2}\right) \leq 19.16\left[\mathrm{~cm}^{-2}\right]$, mean cloud temperatures $37 \mathrm{~K} \leq T_{01} \leq 211 \mathrm{~K}$, and excitation temperatures $423 \mathrm{~K} \leq T_{\mathrm{ex}} \leq 810 \mathrm{~K}$. Using the relation between $N(4) / N(0)$ and $f$, we derive $I_{\mathrm{UV}}$ and $n_{\mathrm{H}}$ not only for our targets, but also for 7 high-latitude FUSE targets. We find that $I_{\mathrm{UV}}$ ranges from 0.4 to 158 and $n_{\mathrm{H}}$ ranges from 6.3 to $2.5 \times 10^{3} \mathrm{~cm}^{-3}$ for our targets. The relation between $n_{\mathrm{H}}$ and $N\left(\mathrm{H}_{2}\right)$ confirms that our results are self-consistent: only clouds with $\log N\left(\mathrm{H}_{2}\right)>18.7$ $\left[\mathrm{cm}^{-2}\right]$ have $n_{\mathrm{H}}>100 \mathrm{~cm}^{-3}$, which means that a mini- 
TABLE 4

Physical PARAmeters ${ }^{a}$

\begin{tabular}{lcccc}
\hline \hline Star & $T_{01}[\mathrm{~K}]$ & $T_{e x}[\mathrm{~K}]$ & $\log f^{b}$ & $\log N(4) / N(0)$ \\
\hline HD 36402 & $54 \pm 4$ & $524 \pm 31$ & $-1.78 \pm 0.07$ & $-4.85 \pm 1.04$ \\
HD 94473 & $67 \pm 4$ & $688 \pm 28$ & $-1.75 \pm 0.07$ & $-3.46 \pm 0.22$ \\
HD 97991 & $37 \pm 8$ & $423 \pm 90$ & $-2.80 \pm 0.16$ & $-3.41 \pm 0.37$ \\
HD 149881 & $97 \pm 5$ & $524 \pm 29$ & $-1.38 \pm 0.06$ & $-4.09 \pm 0.01$ \\
HD 156110 & $69 \pm 4$ & $653 \pm 39$ & $-1.24 \pm 0.04$ & $-4.67 \pm 0.01$ \\
HD 167756 & $46 \pm 3$ & $605 \pm 47$ & $-3.35 \pm 0.05$ & $-2.76 \pm 0.02$ \\
HD 203664 & $79 \pm 6$ & $559 \pm 83$ & $-1.53 \pm 0.08$ & $-4.21 \pm 0.85$ \\
HD 214080 & $59 \pm 4$ & $688 \pm 32$ & $-1.93 \pm 0.07$ & $-4.02 \pm 0.20$ \\
HD 219188 & $211 \pm 14$ & $810 \pm 136$ & $-1.38 \pm 0.09$ & $-3.24 \pm 0.21$ \\
HD 220582 & $82 \pm 5$ & $553 \pm 54$ & $-0.67 \pm 0.06$ & $-3.84 \pm 0.18$ \\
ESO 141-G55 & $144_{-47}^{+184}$ & $406 \pm 317$ & -1.12 & -4.26 \\
HS 0624+6907 & $100_{-18}^{+34}$ & $498 \pm 34$ & -0.76 & -3.90 \\
Mrk 116 & $71_{-12}^{+16}$ & $437 \pm 82$ & -1.06 & -4.06 \\
Mrk 335 & $92_{-14}^{+27}$ & $434 \pm 56$ & -1.32 & -4.21 \\
Mrk 1095 & $121_{-39}^{+107}$ & $501 \pm 129$ & -1.90 & -3.34 \\
NGC 7469 & $71_{-11}^{+16}$ & $389 \pm 35$ & -0.71 & -4.51 \\
VII Zw 118 & $108_{-24}^{+48}$ & $552 \pm 80$ & -1.43 & -3.98 \\
\hline
\end{tabular}

${ }^{a}$ Quoted errors are calculated from the uncertainties shown in Table 3.

${ }^{b} f=\frac{2 N\left(\mathrm{H}_{2}\right)}{\left[N(\mathrm{HI})+2 N\left(\mathrm{H}_{2}\right)\right]}$

${ }^{c}$ High-latitude FUSE data from Gillmon et al. (2006)

mum hydrogen density is required to form a translucent molecular cloud.

\section{ACKNOWLEDGEMENTS}

The ORFEUS Project (Orbiting and Retrievable Far and Extreme Ultraviolet Spectrometers) was a collaboration of the Institute for Astronomy and Astrophysics at the University of Tübingen, the Space Astrophysics Group of the University of California, Berkeley, and the Landessternwarte Heidelberg.

The curve-fitting program SPECFIT runs in the IRAF environment. The Image Reduction and Analysis Facility is distributed by the National Optical Astronomy Observatories, which is supported by the Association of Universities for Research in Astronomy (AURA), Inc., under cooperative agreement with the National Science Foundation.

D.-H. Lee was supported in part by MEST STSAT-3 MIRIS program.

\section{REFERENCES}

Albert, C. E., 1983, Neutral interstellar gas in the lower galactic halo, ApJ, 272, 509

Black, J. H., van Dishoeck, E. F., \& Ewine F., 1987, Fluorescent excitation of interstellar $\mathrm{H} 2$, ApJ, 322, 412
Browning, M. K., Tumlinson, J., \& Shull, J. M., 2003, Inferring Physical Conditions in Interstellar Clouds of $\mathrm{H}_{2}$, ApJ, 582, 810

Diplas, A. \& Savage, B. D., 1994a, An IUE survey of interstellar H I LY alpha absorption. 1: Column densities ApJS, 93, 211

Dixon, W. V., Hurwitz, M., \& Bowyer, S., 1998, ORFEUSI Observations of Molecular Hydrogen in the Galactic Disk, ApJ, 492, 569

Dixon, W. V., 2000, (private communication)

ESA 1997, The Hipparcos and Tycho Catalogues, ESA SP1200

Fruscione, A., Hawkins, I., Jelinsky, P., \& Wiercigroch, A., 1994, The distribution of neutral hydrogen in the interstellar medium. 1: The data, ApJS, 94, 127

Gillmon, K., Shull, J. M., Tumlinson, J., \& Danforth, C., 2006, A FUSE Survey of Interstellar Molecular Hydrogen toward High-Latitude AGNs, ApJ, 636, 891

Howarth, I. D., Siebert, K. W., Hussain, G. A. J., \& Prinja, R. K., 1997, Cross-correlation characteristics of OB stars from IUE spectroscopy, MNRAS, 284, 265

Hurwitz, M. \& Bowyer, S., 1995, ORFEUS Observations of G191-B2B: Neutral and Ionized Gas in the Local Interstellar Medium, ApJ, 446, 812

Hurwitz, M. et al., 1998, Far-Ultraviolet Performance of the Berkeley Spectrograph during the ORFEUS-SPAS II Mission, ApJ, 500, L1 
TABLE 5

DERIVED PHYSICAL PARAMETERS

\begin{tabular}{|c|c|c|c|}
\hline Object & $\left(\log n_{\mathrm{H}} / I_{\mathrm{UV}}\right)$ & $\log I_{\mathrm{UV}}$ & $\log n_{\mathrm{H}}\left[\mathrm{cm}^{-3}\right.$ \\
\hline HD 36402 & 1.2 & -0.4 & 0.8 \\
\hline HD 94473 & 0.9 & 1.5 & 2.4 \\
\hline HD 97991 & 0.8 & 0.5 & 1.3 \\
\hline HD 149881 & 1.3 & 1.0 & 2.3 \\
\hline HD 156110 & 1.3 & 0.2 & 1.5 \\
\hline HD 167756 & 0.5 & 1.0 & 1.5 \\
\hline HD 203664 & 1.5 & 0.4 & 1.9 \\
\hline HD 214080 & 1.0 & 0.6 & 1.6 \\
\hline HD 219188 & 1.2 & 2.2 & 3.4 \\
\hline HD 220582 & 1.7 & 1.6 & 3.3 \\
\hline ESO 141-G55 & 1.2 & 0.4 & 1.6 \\
\hline HS $0624+6907^{a}$ & 1.6 & 1.0 & 2.6 \\
\hline $\operatorname{Mrk} 116^{a}$ & 1.6 & 0.7 & 2.3 \\
\hline Mrk $335^{a}$ & 1.6 & 0.4 & 2.0 \\
\hline Mrk $1095^{a}$ & 0.6 & 1.4 & 2.0 \\
\hline NGC $7469^{a} 1.5$ & 0.6 & 2.1 & \\
\hline VII Zw $118^{a}$ & 1.5 & 0.5 & 2.0 \\
\hline
\end{tabular}

${ }^{a}$ High-latitude FUSE targets (Gillmon et al. 2006).

Jura, M., 1974, Formation and destruction rates of interstellar $\mathrm{H}_{2}, \mathrm{ApJ}, 191,375$

Keenman, F. P., Duftton, P. L., \& McKeith, C. D., 1982, Atmospheric parameters and chemical compositions of eighteen halo OB stars, MNRAS, 200, 673

Kennedy, D. C., Bates, B., \& Kemp, S. N., 1998, The structure of the interstellar gas towards stars in the globular cluster NGC 6541, A\&A, 336, 315

Kriss, G. A., 1994, in ASP Conf. Ser. 61, Astronomical Data Analysis Software and Systems III, Vol. 3 (San Francisco: ASP), 437

Lee, D.-H., Pak, S., Dixon, W. V. D., \& Dishoeck, E. F., 2007, Ultraviolet Excited High-J Molecular Hydrogen in Photodissociation Regions, ApJ, 655, 940 (Paper I)

Oja, T., 1987, UBV photometry of stars whose positions are accurately known. IV, A\&AS, 68, 211

Penprase, B. E., 1992, Photometric and spectroscopic analysis of high Galactic latitude molecular clouds. I - Distances and extinctions of stars toward 25 selected regions, ApJS, 83, 273

Penprase, B. E., 1993, Photometric and spectroscopic analysis of high galactic latitude molecular clouds. II - Highresolution spectroscopic observations of NA I, CA II, CA I, $\mathrm{CH}$, and $\mathrm{CH}(+1)$, ApJS, 88, 433

Rogerson, Jr., J. B. \& Ewell, Jr., M. W., 1985, Ultraviolet line identifications for tau Scorpii, ApJS, 58, 265

Savage, B. D., Bohlin, R. C., Drake, J. F., \& Budich, W., 1977, A survey of interstellar molecular hydrogen. I, ApJ, 216, 291

Savage, B. D. \& De Boer, K. S., 1981, Ultraviolet absorption by interstellar gas at large distances from the galactic plane, ApJ, 243, 460
Savage, B. D. \& Massa, D., 1987, Highly ionized interstellar gas located in the Galactic disk and halo, ApJ, 314, 380

Sembach, K. R., Danks, A. C., \& Savage, B. D., 1993, Optical studies of interstellar material in low density regions of the Galaxy. I - A survey of interstellar NA I and CA II absorption toward 57 distant stars, A\&AS, 100, 107

Sembach, K. R. \& Danks, A. C., 1994, Optical studies of interstellar material in low density regions of the Galaxy, A\&A, 289, 539

Sembach, K. R., 1998, (private communication)

Sharpless, S., 1974, Rotational velocities in the Orion association, AJ, 79, 1073

Shull, J. M. \& Beckwith, S., 1982, Interstellar molecular hydrogen, ARA\&A, 20, 163

Shull, J. M. et al., 2000, Far Ultraviolet Spectroscopic Explorer Observations of Diffuse Interstellar Molecular Hydrogen, ApJ, 538, L73

Shull, J. M. et al., 2005, Axial Rotation in the Later B-Type Emission-Line Stars, ApJ, submitted

Slettebak, A., 1966, Axial Rotation in the Later B-Type Emission-Line Stars, ApJ, 145, 121

Snow, T. P. \& Jenkins, E. B., 1977, A catalog of 0.2 A resolution far-ultraviolet stellar spectra measured with Copernicus, ApJS, 33, 269

Sonnentrucker, P., Friedman, S. D., Welty, D. E., York, D. G., \& Snow, T. P., 2003, Abundances and Physical Conditions in the Interstellar Gas toward HD 185418, ApJ, 596, 350

Spitzer, L. \& Jenkins, E. B., 1975, Ultraviolet studies of the interstellar gas, ARA\&A, 13, 133 
Spitzer, L. \& Fitzpatrick, E. L., 1995, Composition of interstellar clouds in the disk and halo. 3: HD 149881, ApJ, 445, 196

Wakker, B. P. 2001, Distances and Metallicities of Highand Intermediate-Velocity Clouds, ApJS, 136, 463

Walborn, N. R., Lennon, D. J., Haser, S. M., Kudritzki, R. -P., \& Voels, S. A., 1995, The physics of massive OB stars in different parent galaxies. 1: Ultraviolet and optical spectral morphology in the Magellanic Clouds, PASP, 107, 104

Welsh, B. Y., Sasseen, T., Craig, N., Jelinsky, S., \& Albert, C. E., 1997, A Minisurvey of Interstellar Titanium from the Southern Hemisphere, ApJS, 112, 507

Welty, D. E., 2007, Monitoring the Variable Interstellar Absorption toward HD 219188 with Hubble Space Telescope STIS, ApJ, 668, 1012 